\title{
Power Laws, Prices and National Accounts: Are There Any Irregularities?
}

\author{
By CiPRIAN PATER AND DENi MAZREKAJ*
}

Many economic regularities have been found to adhere to power laws. In this paper, we apply Benford's law to consumer price index data from Norway and Zipf's law on a Norwegian report about the history of Norwegian national accounts. Norway is a particularly interesting country to study as it scores among the highest-ranked countries on data quality. We find that the consumer price index adheres to Benford's law, showing high data quality. On the other hand, our results do indicate that the report does not adhere to Zipf's law.

\footnotetext{
*Ciprian Pater: Department of Planning, Administration and Social Sciences, Volda University College, Joplassvegen 116103 Volda, Norway (email: ciprianp@student.hivolda.no); Deni Mazrekaj: Department of Sociology, University of Oxford, Park End Street 42-43, OX1 1JD Oxford, United Kingdom (e-mail: deni.mazrekaj@ sociology.ox.ac.uk); Leuven Economics of Education Research (LEER), KU Leuven, Naamsestraat 69,

3000 Leuven, Belgium (e-mail: deni.mazrekaj@kuleuven.be); The authors declare that they have no relevant or material financial interests that relate to the research described in this paper.
}

Keywords: Power Law; Benford's Law; Zipf's Law; Consumer Price Index; National Accounts. 


\section{Introduction}

A remarkable number of phenomena have been found to follow a power law of the type $Y=k X^{\alpha}$, where $Y$ and $X$ are variables of interest, $\alpha$ is a the power law exponent, and $k$ is a constant (Gabaix, 2009). Two of the most used power laws are Benford's law and Zipf's law. Benford's law (Benford, 1938), first discovered by Newcomb (1881), states that the frequency with which the first digit is 1 is $30.1 \%$, the first is 2 is $17.6 \%$ etc. declining to the first digit being 9 only $4.6 \%$ of the time. Benford's law has been mainly used to detect fraud, claiming that a deviation from Benford's law in real number datasets signifies tampering with the data at hand. For instance, Nigrini (1996) used Benford's law to expose tax fraud, whereas Koch and Okamura (2020) applied Benford's law to investigate whether China manipulated the number of infected persons and the number of deaths during the current COVID-19 pandemic. Another common use of Benford's law is to measure the quality of data, for instance in survey research (Judge \& Schechter, 2009).

Whereas Benford's law is mostly used to analyze numbers, Zipf's law was found to apply for texts. Namely, Zipf's law (Zipf, 1949), first discoved by Auerbach (1913), states that the frequency of a word is proportional to the inverse of its rank. For instance, if the most common word occurs 1,000 times in a text, the second most common word occurs 500 times, the third most common word 333 times etc. Zipf's law has been found across different languages, literary styles, and time periods (Bentz, Verkerk, Kiela, \& Buttery, 2015). Interestingly, Zipf's law has been found even for timbres of sounds (Haro, Serrà, Herrera, \& Corral, 2012), and dolphin whistles. Other more numerical approaches include city size (Arshad, Hu, \& Ashraf, 2018), firm size (Axtell, 2001), and genes expression (Furusawa \& Kaneko, 2003).

In this paper, we apply power laws to prices and national accounts texts in Norway. Specifically, we analyze the Consumer Price Index (CPI) using Benford's law, and a Statistics Norway report about the history of national accounts using Zipf's law. We find that Norwegian CPI data adhere well to Benford's law. On the other hand, the text does not appear to adhere to the Zipf's law.

In the remainder of the paper, we first briefly explain the data sources. Then, we formulate Benford's and Zipf's laws and describe the testing procedures. Finally, we apply the laws on our data and formulate conclusions. 


\section{Data}

We apply power laws to Norway and obtain data from Statistics Norway. Norway is particularly interesting as it is ranked in the top 5 of countries with the highest standard of economic reporting on the Global GDP Data Quality Rating (World Economics Research, 2020). For Benford's law, we use monthly consumer price index data for each good starting from January 2012 until August 2020, totalling 10,788 observations (Statistics Norway, 2020). We removed observations that started with 0. Our final sample includes 9,164 observations. For Zipf's law, we use the official report by Statistics Norway about the history of national accounts, called "History of national accounts in Norway" (Flottum, Halvorsen, Simpson, \& Skoglund, 2012). This report was published in English.

\section{Testing for Benford's Law}

Benford's law can be formulated as follows (Koch \& Okamura, 2020):

$$
P(d)=\log _{10} \frac{(1+d)}{d} \text { for } d \in(1, \ldots, 9)
$$

We test for Benford's law using the commonly applied Chi-Square test of Goodness of Fit:

$$
D^{2}=n \sum_{d=1}^{9} \frac{\left(h_{d}-p_{d}\right)^{2}}{p_{d}}
$$

Where $n$ is the number of observations, $h$ is the observed frequency of each digit, and $p$ is the Benford's Law distribution presented in Table 1.

TABLE 1: BENFORD's LAW DISTRIBUTION

\begin{tabular}{lccccccccc}
\hline \hline First digit & 1 & 2 & 3 & 4 & 5 & 6 & 7 & 8 & 9 \\
Benford distribution probability & .301 & .176 & .125 & .097 & .079 & .067 & .058 & .051 & .046 \\
\hline
\end{tabular}

However, the Chi-Square test is very sensitive in large samples, rejecting even very small differences between the observed and the expected distributions (Koch \& Okamura, 2020). Therefore, we also compute the Mean Standard Deviation (MAD) which is not sensitive to sample size (Silva \& Filho, 2020). 


\section{Testing for Zipf's Law}

Zipf's law essentially follows a Pareto distribution with exponent -1 . It can be formulated as follows:

$$
W=k R^{\alpha} \text { with } \alpha=-1
$$

where $W$ is the word within a text and $\mathrm{R}$ is the rank of this word, signifying the rank the word takes in the entire word frequency distribution. Thus, the most common word in the text takes the rank 1, the second most common word rank 2 etc. To test for Zipf's law, earlier studies have often used either a descriptive analysis in which log of the variable is plotted against log of the rank and it is observed how much the real data deviate from the 45 degress line. A more quantitative approach tests for this relationship as follows:

$$
\log (W)=\log (k)-\alpha \log (R)
$$

If the parameter of interest $\alpha$ is either exactly 1 or close to 1 , it is concluded that a text adheres to the Zipf's law. However, Urzua (2011) shows that this approach is erroneous because OLS estimators are not efficient as rank is an integer, and the intercept is not a nuisance parameter in the regression. Therefore, we use an adapted version of the Lagrange Multiplier (LM) test (Urzua, 2020).

\section{Results}

Figure 1 shows the observed distribution of first digits and the expected distribution if Benford's law holds. We find that the observed distribution closely approximates the expected distribution, suggesting that the Benford's law holds. Nonetheless, we also formally test for Benford's law using the Chi-Square test of Goodness of Fit. The Chi-Square test does not support Benford's distribution for any of the digits after 2 ( $p$-value is lower than .05). However, as explained above, the ChiSquare test is very sensitive to large samples as it tends to reject even very small differences. Therefore, we also compute the Mean Absolute Deviation, which is not sensitive to sample size. The MADs are well above the threshold of 0.015 for all digits, suggesting that the CPI adheres to Benford's law.

Figure 2 shows the observed distribution of word frequencies and the expected distribution if Zipf's law holds. It appears from the figure that the text does not adhere to the Zipf's law. We also formally test this using the Urzua test explained above. We find a $p$-value of close to zero indicating that the Zipf's law indeed does not hold in this text. 


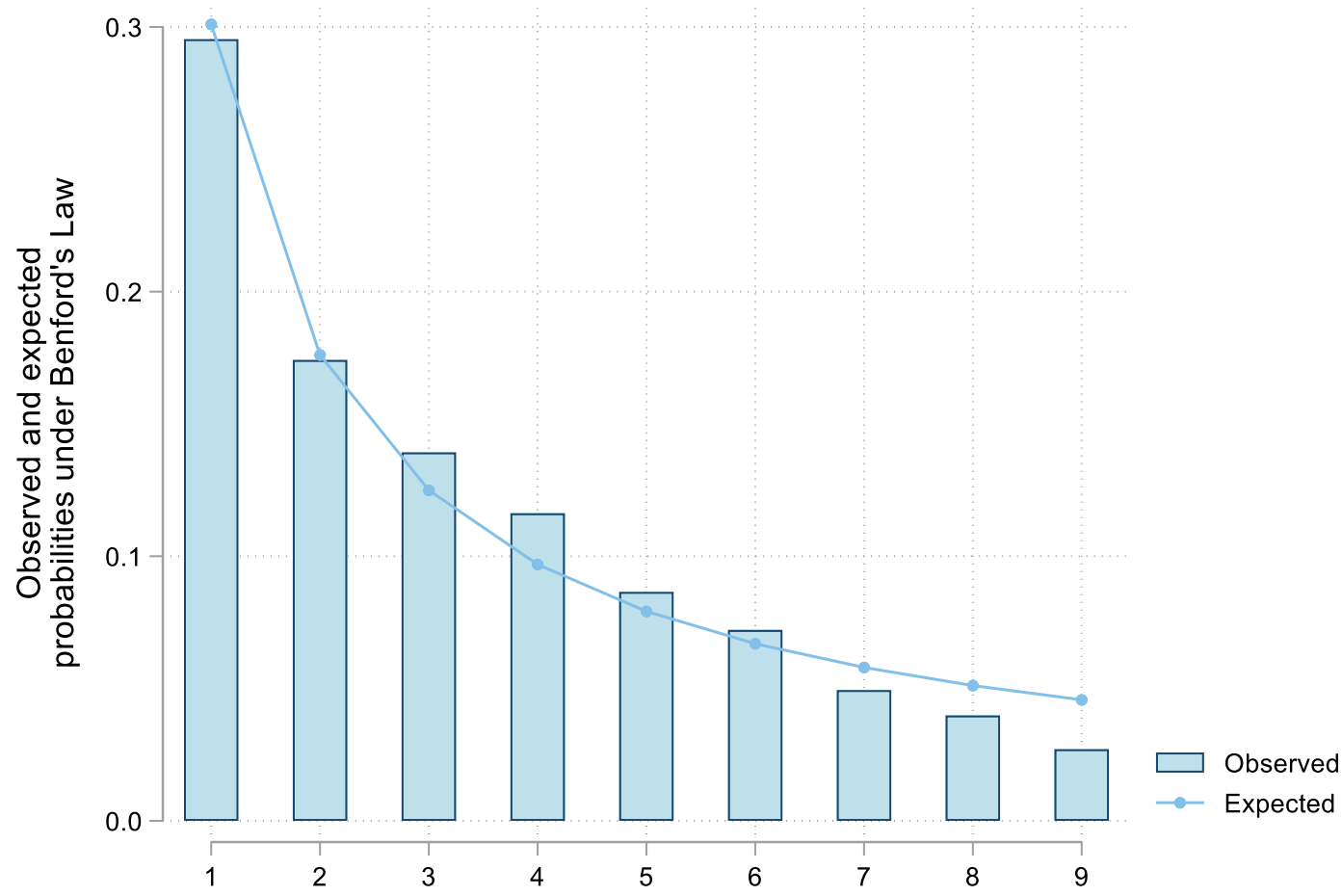

Figure 1: BENFORD’s LAW APPLIED to CONSUMER PRICE INDEX

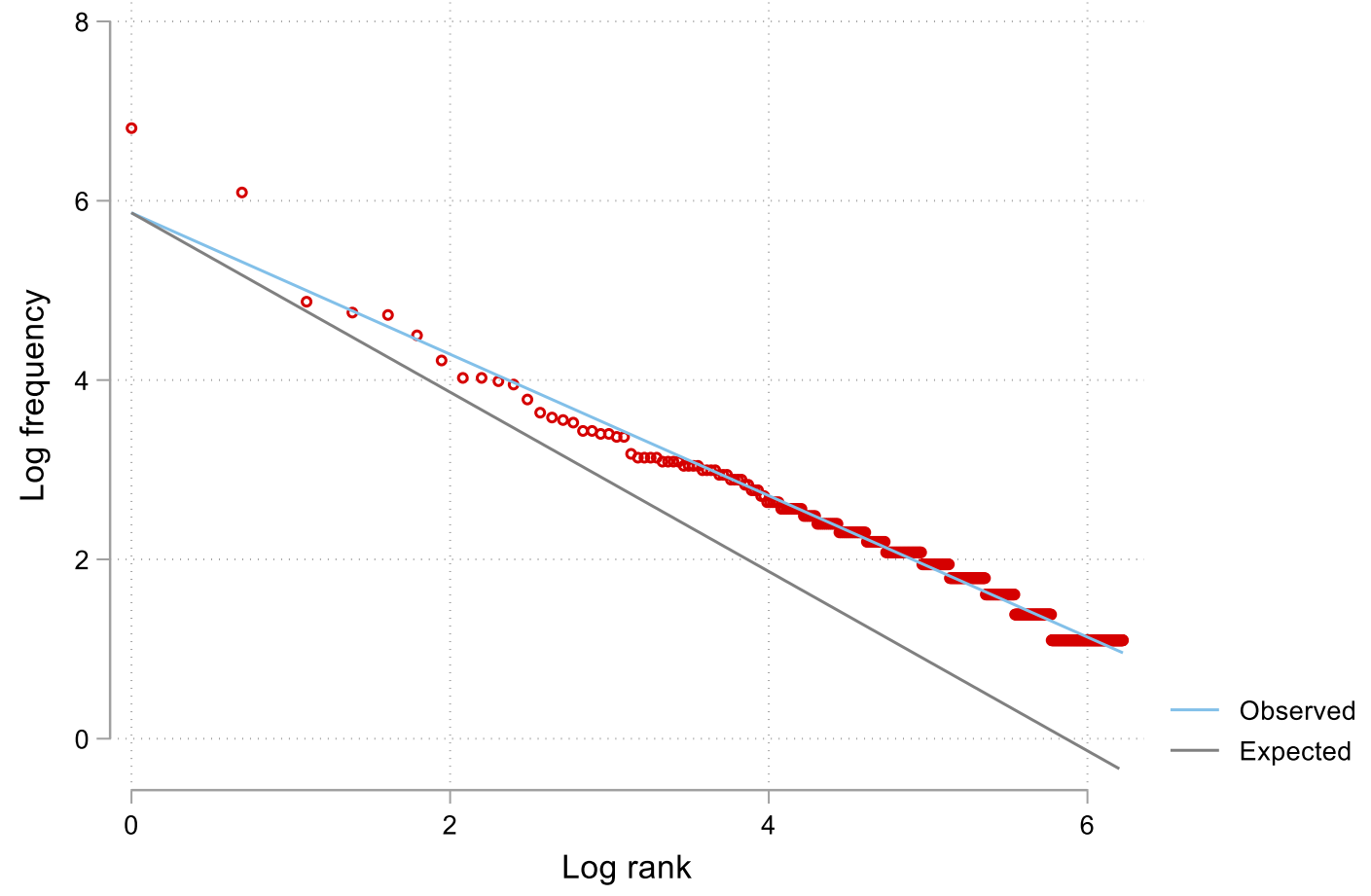

Figure 2: ZiPF's LAW APPLIED to NATIONAL ACCOUNTS TeXT 


\section{Conclusion}

Using data on the consumer price index In Norway, we found that that the CPI adheres to Benford's law. This indicates that Norway does indeed publish high quality statistics on inflation. On the other hand, we found no evidence that a report from Statistics Norway adheres to the Zipf's law. This contradicts previous studies that claim that Zipf's law holds for all texts. Further research should investigate the mechanism why Zipf's law does not hold in this context and how this irregularity may be connected to the current socio-economic environment.

\section{References}

Arshad, S., Hu, S., \& Ashraf, B. N. (2018). Zipf's law and city size distribution: A survey of the literature and future research agenda. Physica A, 492, 75-92.

Auerbach, F. (1913). Das Gesetz der Bevölkerungskonzentration. Petermanns Geographische Mitteilungen, 59, 74-76.

Axtell, R. L. (2001). Zipf Distribution of U.S. Firm Sizes. Science, 293, 1818-1820.

Benford, F. (1938). The Law of Anomalous Numbers. Proceedings of the American Philosophical Society, 78(4), 551-572.

Bentz, C., Verkerk, A., Kiela, D., \& Buttery, P. (2015). Adaptive Communication: Languages with More Non-Native Speakers Tend to Have Fewer Word Forms. PLoS ONE, 10(6), 1-23.

Flottum, E. J., Halvorsen, T., Simpson, L. H., \& Skoglund, T. (2012, March). History of national accounts in Norway. Retrieved from Statistics Norway: https://www.ssb.no/en/nasjonalregnskap-og-konjunkturer/artikler-ogpublikasjoner/history-of-national-accounts-innorway\#: :text=The\%20National\%20Accounts\%20Unit\%20was,\%2D1939\%20and\%201 946\%2D1951.\&text=The\%20first\%20estimates\%20from\%20quarterly,temporarily

Furusawa, C., \& Kaneko, K. (2003). Zipf's Law in Gene Expression. Physical Review Letters, 90(8), 1-4.

Gabaix, X. (2009). Power Laws in Economics and Finance. Annual Review of Economics, 1, 255293.

Haro, M., Serrà, J., Herrera, P., \& Corral, A. (2012). Zipf's Law in Short-Time Timbral Codings of Speech, Music, and Environmental Sound Signals. PLoS ONE, 7(3), 1-10. 
Judge, G., \& Schechter, L. (2009). Detecting Problems in Survey Data Using Benford's Law. Journal of Human Resources, 44, 1-24.

Koch, C., \& Okamura, K. (2020). Benford's Law and COVID-19 reporting. Economics Letters, $196,1-4$.

Newcomb, S. (1881). Note on the Frequency of Use of the Different Digits in Natural Numbers. American Journal of Mathematics, 4(1), 39-40.

Nigrini, M. J. (1996). A Taxpayer Compliance Application of Benford's Law. Journal of the American Taxation Association, 18(1), 72-91.

Silva, L., \& Filho, D. F. (2020). Using Benford's law to assess the quality of COVID-19 register data in Brazil. Journal of Public Health, 1-4.

Statistics Norway. (2020, October). Consumer price index. Retrieved from Statistics Norway: https://www.ssb.no/en/statbank/list/kpi/

Urzua, C. M. (2011). Testing for Zipf's law: A common pitfall. Economics Letters, 112, 254-255.

Urzua, C. M. (2020). A simple test for power-law behavior. Stata Journal, 20(3), 604-612.

Zipf, G. K. (1949). Human Behavior and the Principle of Least Effort: An Introduction to Human Ecology. Cambridge, Massachusetts: Addison-Wesley. 